\title{
Evaluation of transforming growth factor beta 1 in dogs with osteoarthritis
}

\author{
Stephan Neumann* and Sarah Lauenstein-Bosse \\ Institute of Veterinary Medicine, Georg-August University Göttingen, Burckhardtweg 2, D-37077 Göttingen, \\ Germany
}

\begin{abstract}
Osteoarthritis is a common problem in daily veterinary practice with insufficient knowledge about disease mechanism. Because fibrosis is a part of the alteration in the effected joints, we investigated the transforming growth factor beta 1 (TGF- $\beta 1$ ) as an important regulation molecule of tissue fibrosis. We chose Osteoarthritis following a cruciate ligament rupture (CLR) because it is a common model of osteoarthritis. A total of 13 healthy dogs and 38 dogs suffering from CLR were included in this prospective study. The concentration of TGF- $\beta 1$ was measured in synovial fluid using the "Quantikine Human TGF- $\beta 1$ Immunoassay" (Fa. R \& D Systems, Minneapolis, USA). There was a significant difference in the TGF- $\beta 1$ concentration of the synovial fluid of healthy compared to affected patients $(\mathrm{p}<0.001)$. The synovial TGF- $\beta 1$ concentration also correlated significantly $(p=0.0147)$ with the synovial viscosity of the affected patients. No significant correlations could be observed to duration of disease, severity of lameness and degree of joint swelling, but also to joint capsule sickness, osteophyte development and degeneration of joint cartilage. The results of our study show an increased TGF- $\beta 1$ concentration in knees affected with osteoarthritis as consequence of CLR. So we can conclude that TGF- $\beta 1$ takes part at the osteoarthritic remodelling process, but different phases of the remodelling process cannot be distinguished by the measurement of TGF- $\beta 1$.
\end{abstract}

Keywords: Dog, Osteoarthritis, TGF- $\beta 1$.

\section{Introduction}

Osteoarthritis has an occurrence of more than $20 \%$ in dogs older than one year and $80 \%$ in geriatric dogs older than eight years (Johnston, 1997). The high prevalence of this condition in canines demonstrates its relevance and the need of further research into this topic. Cruciate ligament rupture (CLR) is the main cause of osteoarthritic changes in the canine knee joint (Pond and Campbell, 1972; Hayashi et al., 2004). The result of the damage of the cruciate ligament (CL) is always the development of osteoarthritis (Tirgari and Vaughan, 1975).

Osteoarthritis is defined as a primary, noninflammatory, painful, degenerative disease of the joint, that leads to progressive changes in the structure of the cartilage and bone after a prolonged period of continuous inappropriate biomechanical stress of the joint (Brandt et al., 2006).

The joint capsule also experiences a fibrotic redevelopment process which becomes clinically apparent through the restricted mobility of the joint (Solimeno et al., 2010). Fibrosis is characterized by a pathologic increase of connective tissue, caused by chronic damage (Wynn, 2008). This goes along with proliferation and activation of fibroblasts that synthesise high amounts of altered extracellular matrix (Krieg et al., 2007). Individual components of the extracellular matrix, such as collagen and fibronectin, are increasingly produced during fibrosis (Wynn, 2008). Furthermore, the degradation of the extracellular matrix is reduced through the inactivation of matrix metalloproteinases (MMPs) (Eddy, 1996).

Cytokines play a key role in fibrosis (Borthwick et al., 2013). Especially the cytokine transforming growth factor beta 1 (TGF- $\beta 1$ ) plays a decisive role in the fibrotic changes of tissue (Murakami et al., 1995; Branton and Kopp, 1999; Zeichen et al., 2008). Through the overexpression of TGF- $\beta 1$ it comes to an increased synthesis of collagen type 1 and 3 , as well as fibronectin (Ignotz and Massagué, 1986; Varga et al., 1987; Lafyatis et al., 1989). TGF- $\beta 1$ diminishes the expression of MMPs and inducing an accumulation of extracellular matrix (Edwards et al., 1987).

Thus, the aim of this study was to evaluate the relevance and possible diagnostic utility of TGF- $\beta 1$ in canine osteoarthritis. Therefore, TGF- $\beta 1$ was measured in synovial fluid of dogs with osteoarthritis as a consequence of CLR and a possible connection between the cytokine levels, morphologic and functional alterations of the joint was analysed.

\section{Animals and Samples}

\section{Materials and Methods}

A total of 51 dogs, patients of the Small Animal Clinic of the University of Göttingen, were included in this prospective study. Thirteen patients were free of orthopaedic conditions of the knee and 38 dogs had a 
CLR. The 13 dogs without clinical-orthopaedic reports were used as a control group. These dogs were euthanized due to different medical conditions. Post mortem, synovial fluid of one knee joint was drawn, medio-lateral and cranio-caudal $\mathrm{x}$-rays of the punctured knee joint were made and the knee was inspected. The examination of the knee joint showed no pathologic changes and the macroscopic examination of the synovial fluid was normal.

The presumptive diagnosis of CLR in the 38 diseased patients was made by palpatory examination via the "Anterior-Drawer-Test" or the "Tibial-CompressionTest" (Johnson and Johnson, 1993). A definitive diagnosis was made during the intraoperative evaluation of the ligament. The presence of other pathologies was ruled out by a detailed anamnesis, clinical examination, complete blood cell count (CBC) and serum chemistry. The synovial fluid was drawn under anaesthesia by puncturing the unopened knee joint, which had been previously prepared for surgery of the CLR. After its macroscopic evaluation, the synovial fluid of both groups was centrifuged and the supernatant extracted and frozen at $\leq-70{ }^{\circ} \mathrm{C}$ until needed for the analysis of TGF- $\beta 1$. The affected patients were divided into different groups according to the disease severity that was determined through the orthopaedic examination, the evaluation of the synovial fluid and the intraoperative inspection of the knee joint. The whole evaluation was performed by the same veterinary surgeon. All samples were acquired with the owner's consent, and the procedure was in accordance with the German Protection of Animals Act and carried out under the supervision of the animal welfare officer of the Faculty of Agriculture, University of Goettingen. TGF- $\beta 1$ ELISA

The "Quantikine Human TGF- $\beta 1$ Immunoassay" (Fa. R \& D Systems, Minneapolis, USA) is a SandwichELISA that can measure TGF- $\beta 1$ in serum. The antibodies bound to the plate are monoclonal for TGF$\beta 1$. There is no information from the manufacturer about its applicability for synovial fluid, but this ELISA has already been used successfully for the examination of synovial fluid in humans (Pagura et al., 2005). The assessment of TGF- $\beta 1$ was done according to the manufacturer's instructions.

In summary, the diluted standards, controls and samples were applied in triplicates to the coated microtiter plate. The prepared plates were incubated for two hours and then rinsed to remove any unbound material. A conjugate of TGF- $\beta 1$ antibodies was applied to the plates and incubated for two hours followed by rinsing. A substrate solution was added and the plate was incubated for 30 minutes. During this time, the substrate solution developed colour depending on its TGF- $\beta 1$ content. Applying a stop solution terminated this process. The optical density of the developed colour was determined photometrically with a TECAN GENios Pro (Fa. TECAN Austria $\mathrm{GmbH}$, Grödig, Austria) at a wavelength of $450 \mathrm{~nm}$ and a correcting wavelength of $570 \mathrm{~nm}$. According to the manufacturer the smallest detectable dose of human TGF- $\beta 1$ is $4.61 \mathrm{pg} / \mathrm{mL}$.

\section{Statistical analysis}

A Mann-Whitney U-Test was used to determine differences between the TGF- $\beta 1$ concentrations in the synovial fluid of healthy and affected patients. A correlation between the synovial TGF- $\beta 1$ levels and the different characteristics previously described was determined using a Spearman's rank correlation coefficient. The correlation was analysed among each characteristic. All statistical analysis was done in IBM SPSS Statistics 22.0 (IBM Corp. Released 2011. IBM SPSS Statistics for Windows, Version 22.0. Armonk, NA:IBM Corp.). The level of significance was $\mathrm{p}=0.05$. TGF- $\beta 1$ concentration above $3 \mathrm{ng} / \mathrm{dl}$ were set as $>3$.

\section{Results}

The TGF- $\beta 1$ concentration in the synovial fluid of healthy patients was between 0.78 and $1.39 \mathrm{ng} / \mathrm{ml}$ (median: $1.20 \mathrm{ng} / \mathrm{ml}$ ). Dogs with CLR showed a median concentration of TGF- $\beta 1$ of $2.13 \mathrm{ng} / \mathrm{ml}$ (range, $0.92-\geq 3 \mathrm{ng} / \mathrm{ml}$ ) in their synovial fluid. There was a significant difference $(\mathrm{p}<0.001)$ between the synovial fluid TGF- $\beta 1$ concentrations of healthy and affected dogs (Fig. 1). According to the duration of lameness four groups were. Patients with lameness for 1 to 15 days were assigned to group 1. Patients with lameness for 16 to 30 days were assigned to group 2 and with lameness for 31 to 45 days were assigned to group 3 . All patients with lameness for longer than 45 days were assigned to group 4. There was no significant correlation between the TGF- $\beta 1$ concentrations in synovial fluid and the duration of lameness ( $>0.05)$ (Table 1).

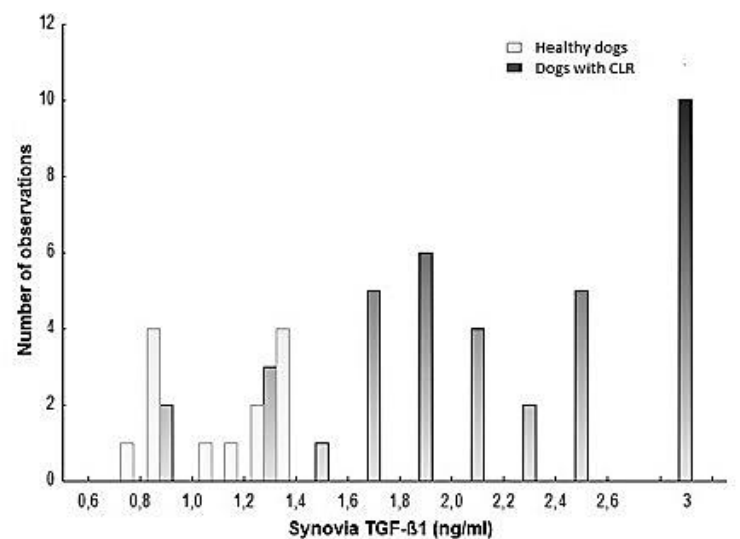

Fig. 1. Stacked bar chart of the TGF- $\beta 1$ concentrations in the synovial fluid of healthy dogs $(n=13)$ and dogs with CLR (n $=38$ ). The synovial fluid of dogs with CLR had a significantly higher TGF- $\beta 1$ concentration than the synovial fluid of healthy dogs $(\mathrm{p}<0.001)$. Dogs with TGF- $\beta 1$ concentrations $\geq 3 \mathrm{ng} / \mathrm{ml}$ were set at $3 \mathrm{ng} / \mathrm{ml}$. 
Table 1. TGF- $\beta 1$ and orthopedic investigation.

\begin{tabular}{lccc}
\hline $\begin{array}{l}\text { Duration of } \\
\text { lameness }\end{array}$ & $\begin{array}{c}\text { No. of } \\
\text { patients }\end{array}$ & $\begin{array}{c}\text { TGF- } \beta 1 \\
\text { (ng/dl) } \\
\text { median }\end{array}$ & Min-Max \\
\hline 1-15 days & 21 & 1.98 & $0.98->3$ \\
\hline 16-30 days & 6 & 2.78 & $1.52->3$ \\
\hline 31-45 days & 2 & 2.5 & $1.99->3$ \\
\hline 45 days & 9 & 2.11 & $0.92->3$ \\
\hline Degree of lameness & 5 & 2.11 & $1.64->3$ \\
\hline grade 1 & 9 & 2.31 & $1.4->3$ \\
\hline grade 2 & 14 & 2.33 & $0.92->3$ \\
\hline grade 3 & 10 & 1.94 & $0.98->3$ \\
\hline grade 4 & 8 & & \\
\hline Degree of joint swelling & 15 & 2.05 & $1.4->3$ \\
\hline grade 0 & 7 & 2.55 & $1.64->3$ \\
\hline grade 1 & 8 & 2.18 & $0.92->3$ \\
\hline grade 2 & 2.08 & $0.98->3$ \\
\hline grade 3 & &
\end{tabular}

Affected dogs were also grouped in accordance to the severity of their lameness following the classification scheme of Arnoczky and Tarvin (Arnoczky and Tarvin, 1981). Patients classified as group 1 showed very slight signs of lameness of the affected limb. Patients assigned to group 2 showed clear signs of lameness but still put pressure on the affected limb, while patients in group 3 rarely put weight on the affected limb. Patients that avoided completely the use of the affected limb were classified as group 4. There was no significant correlation between the TGF- $\beta 1$ concentrations in synovial fluid and the severity of lameness $(p>0.05)$ (Table 1).

Moreover, patients were grouped according to the degree of periarticular swelling of the joint. Dogs classified as group 0 did not present any swelling. In group 1 a mild periarticular swelling could be detected by palpation.

A moderate periarticular swelling was allocated into group 2 and a severe periarticular swelling was classified as group 3. There was no significant correlation between the TGF- $\beta 1$ concentrations in synovial fluid and the severity of lameness $(p>0.05)$ (Table 1).

In summery no significant correlation between the TGF- 31 synovia concentrations and clinic symptoms of the investigated dogs could be found.
Four groups were established based on the viscosity of the synovial fluid. Synovia with a very high viscosity and ropy to a length $>5 \mathrm{~cm}$ was classified as group 0 . In group 1 the synovia had a high viscosity, forming ropy of $2-5 \mathrm{~cm}$.

Group 2 presented a reduced viscosity with $<2 \mathrm{~cm}$ ropy and in group 3 the patients synovia showed a very low, liquid viscosity that did not ropy.

Regarding the synovial viscosity, a significant positive correlation was detected between group 1 (median: $1.96 \mathrm{ng} / \mathrm{ml}$ ) and group 2 (median: $2.55 \mathrm{ng} / \mathrm{ml}$ ) in the synovial TGF- $\beta 1$ concentrations $(p=0.0147)$ (Fig. 2$)$.

So TGF- 11 synovia concentration increases in cases of reduced synovia viscosity.

The intraoperative examination included the following parameters: changes of the joint capsule and the synovial membrane, osteophyte formation and degeneration of the joint cartilage.

Dogs were also separated into different groups based on changes of the joint capsule and the synovial membrane. Patients with a thin joint capsule and a moist, smooth and shiny synovial membrane were classified as group 0 .

Group 1 patients had a slightly thickened joint capsule and a beginning hyperaemia of the synovial membrane. Patients with a moderate to highly thickened joint capsule as well as a hyperaemic synovial membrane were allocated to group 2. All patients in group 3 showed a fibrotic joint capsule and a hyperaemic synovial membrane. There was no significant correlation between the groups according to the joint capsule $(\mathrm{p}=0.794)$ (Table 2$)$.

In all patients the formation of osteophytes was documented. Patients lacking the formation of osteophytes were allocated to group 0 . In group 1 patients showed a slight development of flat osteophytes. Dogs with a moderate to high osteophyte formation were allocated to group 2 and patients presenting an osteophyte wall were put into group 3 . The correlation between the different groups based on the osteophyte formation was not significant $(\mathrm{p}=0.9958)$ (Table 2).

In terms of degradation of the joint cartilage, patients were allocated to group 0 if they had a bluish white, smooth and moist cartilage. A slightly yellow to brown cartilage with the onset of roughness was classified as group 1 and patients with a spotty, fraying cartilage were allocated to group 2. Patients with an ulceration of the cartilage and subchondral bone exposure are put into group 3. No significant correlation between the different groups could be observed $(\mathrm{p}=0.422)$ (Table 2$)$. In summery the TGF- $\$ 1$ synovia concentration is not directly associated with different degrees of joint capsule fibrosis, osteophyte development and cartilage degeneration. 


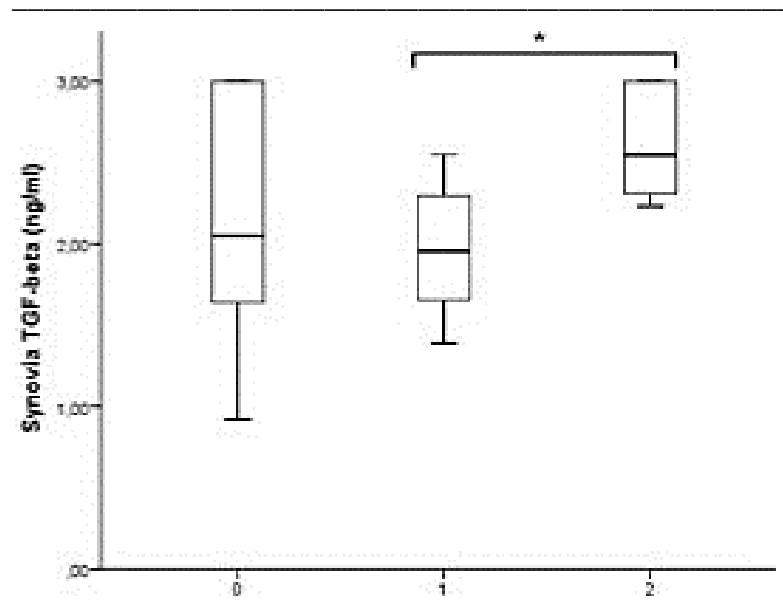

Fig. 2. Box plot of the TGF- $\beta 1$ concentration in synovial fluid of dogs with cruciate ligament rupture, allocated to degrees 0 to 3 based on the viscosity of the synovial fluid $(n=26$, degree $0 ; \mathrm{n}=8$, degree $1 ; \mathrm{n}=6$, degree $2 ; \mathrm{n}=0$, degree 3 ). In grade 1 and grade 2 , a significant correlation with the TGF- $\beta 1$ concentration in the synovial fluid was detected using Spearman's rank correlation test $(\mathrm{p}=0.0147)$. Observations among other groups had no significant correlation using the same test $(p>0.05)$. Dogs with TGF- $\beta 1$ concentrations in synovial fluid of $\geq 3 \mathrm{ng} / \mathrm{m}$ were set at $3 \mathrm{ng} / \mathrm{ml}$. (*p < 0.05 ).

Table 2. TGF- $\beta 1$ and surgical investigation.

\begin{tabular}{lccc}
\hline $\begin{array}{l}\text { Thickness of } \\
\text { joint capsule }\end{array}$ & $\begin{array}{c}\text { No. of } \\
\text { patients }\end{array}$ & $\begin{array}{c}\text { TGF- } \beta 1 \mathrm{ng} / \mathrm{dl} \\
\text { median }\end{array}$ & Min-Max \\
\hline group 0 & 16 & 2.08 & $0.98->3$ \\
group 1 & 12 & 1.91 & $1.37->3$ \\
group 2 & 8 & 2.22 & $0.92->3$ \\
\hline group 3 & 2 & 2.66 & $2.31->3$ \\
\hline Osteophyte formation & 17 & 2.05 & $0.92->3$ \\
\hline grade 0 & 9 & 2.15 & $1.40->3$ \\
\hline grade 1 & 10 & 2.16 & $1.37->3$ \\
\hline grade 2 & 2 & 2.08 & $1.84->3$ \\
\hline grade 3 & - & - & - \\
\hline cartilage degeneration & 30 & 2.08 & $0.92->3$ \\
\hline group 0 & 7 & 2.44 & $1.37->3$ \\
\hline group 1 & 1 & 2.31 & - \\
\hline group 2 & - & & - \\
\hline group 3 & & & \\
\hline
\end{tabular}

\section{Discussion}

Due to the clinical relevance of osteoarthritis in veterinary medicine and the variable prognosis depending on the severity of the condition there is an intensive research about the mechanisms of the disease.
Nevertheless, cytokines like TGF- $\beta 1$ that are known to be involved in fibrotic processes, have not been examined sufficiently in veterinary medicine. Especially for dogs literature about this cytokine is scarce. Therefore, we evaluated the concentration of TGF- $\beta 1$ in synovial fluid of dogs with CLR in association with local osteoarthritic changes that usually develop in the presence of this condition. The aim of this study was to analyse the potential role of TGF- $\beta 1$ during the pathogenesis of osteoarthritis.

The cytokine TGF- $\beta 1$ is known to play an important role in the metabolism of bone and cartilage, but also influences the pathogenesis of fibrosis in humans. Bones have the highest TGF- $\beta 1$ concentration within the body (Bonewald and Mundy, 1990). High concentrations can also be detected in all zones of the cartilage (Fukumura et al., 1998). TGF- $\beta 1$ stimulates the growth, proliferation and biological activity of osteoblasts and plays a central role in the growth and differentiation of chondrocytes (Centrella et al., 1988; Frenkel et al., 2000). TGF- $\beta 1$ also has a stimulating effect on the synthesis of extracellular matrix through chondrocytes and influences the homeostasis of the mature joint cartilage (Redini et al., 1988; Yang et al., 2001). On the other hand, osteoclasts are known to be blocked by TGF- $\beta 1$ (Pfeilschifter et al., 1988).

Dogs with a CLR were chosen for this study, as this condition induces abnormal movement and weight distribution consequently leading to osteoarthritis (Hasler et al., 1998). Due to increased irritation the joint cartilage is damaged. The break down products of the cartilage enter the synovial fluid causing inflammation of the synovial membrane, which then becomes hyperplastic. The joint capsule becomes increasingly fibrotic, subsequently decreasing the instability of the joint (Marshall and Olsson, 1971). Subchondral bone sclerosis occurs after ulceration of the cartilage and puts further pressure on the joint. Finally, marginal osteophytes develop.

After a CLR, osteophytes and erosion of the cartilage develop in all compartments of the canine knee joint within eight weeks (Vavken et al., 2013). First damage to the cartilage has already occurred at the time of the CLR diagnosis because the degenerative changes that lead to a CLR also affect other structures of the knee joint. Osteoarthritic changes could be observed in a number of the dogs included in this study. Yet, in 12 patients no changes to the knee joint besides the rupture of the ACL could be detected during surgical examination. A possible reason could be that the changes were only microscopic at the time of surgery due to an early diagnosis and treatment of the CLR. These patients showed lameness for less than 15 days. In our study, dogs with a CLR had significantly higher synovial TGF- $\beta 1$ concentrations than healthy dogs. The human TGF- $\beta 1$ ELISA has never been used for the 
determination of TGF- $\beta 1$ in canine synovia and reference values are not available. In humans with rheumatoid arthritis higher levels of synovial TGF- $\beta$ were found in contrast to patients with osteoarthritis (Fava et al. 1989). A comparison between healthy and osteoarthritic patients was performed and a higher concentration of TGF- $\beta 1$ in patients with osteoarthritis was given (Ping-Ke et al., 1999). In another study, osteoarthritic changes were observed after multiple intra-articular TGF- $\beta 1$ injections (van Beuningen et al., 2000). On the other hand, the administration of TGF$\beta 1$ antibodies into the synovial fluid can in turn prevent osteoarthritis (Wahl et al., 1993). Chondrocytes are known to contain high concentrations of TGF- $\beta 1$ and to influence its levels in the joints (Fukumura et al., 1998). Due to its known fibrotic effect in other organs it is assumed, that this cytokine can be secreted into the synovial fluid in response to biochemical stress within the joint generating fibrosis. When analysing synovial TGF- $\beta 1$ in relation to the synovial viscosity, patients with a reduced synovial viscosity presented higher TGF- $\beta 1$ than patients with a high synovial viscosity. Synovial fluid is known to be produced by the synovial membrane and its primary lubricant molecule is hyaluronan (Ogston and Stanier, 1953). The concentration of this molecule determines the viscosity of the synovia. In an osteoarthritic joint the hyaluronan concentration is lower compared to a normal joint causing a decrease of the synovial viscosity (Belcher $e t$ al., 1997). It has been shown, that hyaluronan has a stimulating and inhibiting effect on cytokines (Stern $e t$ al., 2006). However, the relationship between TGF- $\beta 1$ and hyaluronan or the synovial viscosity are not sufficiently described. In one study, TGF- $\beta 1$ was shown to enhance the production of hyaluronan in the synovial membrane and to probably contribute in the production of hyaluronan in the joint fluid (Tanimoto $e t$ al., 2004). No significant link was found between synovial TGF- $\beta 1$ and changes of the joint capsule and synovial membrane, the formation of osteophytes and the degeneration of the joint cartilage. Other studies found elevated levels of TGF- $\beta 1$ in the joint cartilage of mice with osteoarthritis and that the repeated application of TGF- $\beta 1$ in a healthy mouse joint causes synovial hyperplasia and the onset of osteophyte formation (van Beuningen et al., 1994; Chambers et al., 1997). In another study, a three to four fold increase of TGF- $\beta 1$ was found in the subchondral bone of osteoarthritically impacted joints compared to healthy joints (Mansell and Bailey, 1998). Regarding humans, osteophytes in osteoarthritically impacted joints show a higher TGF- $\beta 1$ expression (Verdier et al., 2005). Higher TGF- $\beta 1$ concentrations have also been detected histologically in the Hoffa-fatty particle after a rupture of the ACL in humans (Murakami et al., 1995).
In Conclusion of our results, an effect of TGF- $\beta 1$ in the pathogenesis of osteoarthritis in dogs is probable. The concentration is elevated after the trauma and was present during the total period of investigation, which was 45 days. So we can further conclude that the alteration in the effected joint which goes throw an osteoarthritis starts directly after the trauma.

\section{Acknowledgements}

This research did not receive any specific grant from funding agencies in the public, commercial, or not-forprofit sectors.

\section{Conflict of interest}

The authors declare that there is no conflict of interest.

\section{References}

Arnoczky, S.P. and Tarvin, G.B. 1981. Physical examination of the musculoskeletal system. Vet. Clin. North Am. Small Anim. Pract. 11, 575-593.

Belcher, C., Yaqub, R., Fawthrop, F., Bayliss, M. and Doherty, M. 1997. Synovial fluid chondroitin and keratan sulphate epitopes, glycosaminoglycans, and hyaluronan in arthritic and normal knees. Ann. Rheum. Dis. 56, 299-307.

Bonewald, L.F. and Mundy, G.R. 1990. Role of transforming growth factor-beta in bone remodeling. Clin. Orthop. Relat. Res. 250, 261-276.

Borthwick, L.A., Wynn, T.A. and Fisher, A.J. 2013. Cytokine mediated tissue fibrosis. Biochim. Biophys. Acta 1832, 1049-1060.

Brandt, K.D., Radin, E.L., Dieppe, P.A. and van de Putte, L. 2006. Yet more evidence that osteoarthritis is not a cartilage disease. Ann. Rheum. Dis. 65, 1261-1264.

Branton, M.H. and Kopp, J.B. 1999. TGF-beta and fibrosis. Microbes Infect. 1, 1349-1365.

Centrella, M., McCarthy, T.L. and Canalis, E. 1988. Skeletal tissue and transforming growth factor beta. FASEB J. Off Publ. Fed. Am. Soc. Exp. Biol. 2, 3066-3073.

Chambers, M.G., Bayliss, M.T. and Mason, R.M. 1997. Chondrocyte cytokine and growth factor expression in murine osteoarthritis. Osteoarthritis Cartilage 5, 301-308.

Eddy, A.A. 1996. Molecular insights into renal interstitial fibrosis. J. Am. Soc. Nephrol. 7(12), 2495-2508.

Edwards, D.R., Murphy, G., Reynolds, J.J., Whitham, S.E., Docherty, A.J., Angel, P. and Heath, J.K. 1987. Transforming growth factor beta modulates the expression of collagenase and metalloproteinase inhibitor. EMBO J. 6, 1899-1904.

Fava, R., Olsen, N., Keski-Oja, J., Moses, H. and Pincus, T. 1989. Active and latent forms of transforming growth factor beta activity in synovial effusions. J. Exp. Med. 169, 291-296. 
Frenkel, S.R., Saadeh, P.B., Mehrara, B.J., Chin, G.S., Steinbrech, D.S., Brent, B., Gittes, G.K. and Longaker, M.T. 2000. Transforming growth factor beta superfamily members: role in cartilage modeling. Plast. Reconstr. Surg. 105, 980-990.

Fukumura, K., Matsunaga, S., Yamamoto, T., Nagamine, T., Ishidou, Y. and Sakou, T. 1998. Immunolocalization of transforming growth factorbeta s and type I and type II receptors in rat articular cartilage. Anticancer Res. 18, 4189-4193.

Hasler, E.M., Herzog, W., Leonard, T.R., Stano, A. and Nguyen, H. 1998. In vivo knee joint loading and kinematics before and after ACL transection in an animal model. J. Biomech. 31, 253-262.

Hayashi, K., Manley, P.A. and Muir, P. 2004. Cranial cruciate ligament pathophysiology in dogs with cruciate disease: a review. J. Am. Anim. Hosp. Assoc. 40, 385-390.

Ignotz, R.A. and Massagué, J. 1986. Transforming growth factor-beta stimulates the expression of fibronectin and collagen and their incorporation into the extracellular matrix. J. Biol. Chem. 261, 4337-4345.

Johnson, J.M. and Johnson, A.L. 1993. Cranial cruciate ligament rupture. Pathogenesis, diagnosis, and postoperative rehabilitation. Vet. Clin. North Am. Small Anim. Pract. 23, 717-733.

Johnston, S.A. 1997. Osteoarthritis. Joint anatomy, physiology, and pathobiology. Vet. Clin. North Am. Small Anim. Pract. 27, 699-723.

Krieg, T., Abraham, D. and Lafyatis, R. 2007. Fibrosis in connective tissue disease: the role of the myofibroblast and fibroblast-epithelial cell interactions. Arthritis Res. Ther. 9(Suppl. 2), S4. doi: 10.1186/ar2188.

Lafyatis, R., Thompson, N.L., Remmers, E.F., Flanders, K.C., Roche, N.S., Kim, S.J., Case, J.P., Sporn, M.B., Roberts, A.B. and Wilder, R.L. 1989. Transforming growth factor-beta production by synovial tissues from rheumatoid patients and streptococcal cell wall arthritic rats. Studies on secretion by synovial fibroblast-like cells and immunohistologic localization. J. Immunol. 143, 1142-1148.

Mansell, J.P. and Bailey, A.J. 1998. Abnormal cancellous bone collagen metabolism in osteoarthritis. J. Clin. Invest. 101, 1596-1603.

Marshall, J.L. and Olsson, S.E. 1971. Instability of the knee. A long-term experimental study in dogs. J. Bone Joint Surg. Am. 53, 1561-1570.

Murakami, S., Muneta, T., Furuya, K., Saito, I., Miyasaka, N. and Yamamoto, H. 1995. Immunohistologic analysis of synovium in infrapatellar fat pad after anterior cruciate ligament injury. Am. J. Sports Med. 23, 763-768.
Ogston, A.G. and Stanier, J.E. 1953. The physiological function of hyaluronic acid in synovial fluid; viscous, elastic and lubricant properties. J. Physiol. 119, 244-252.

Pagura, S.M.C., Thomas, S.G., Woodhouse, L.J., Ezzat, S. and Marks, P. 2005. Circulating and synovial levels of IGF-I, cytokines, physical function and anthropometry differ in women awaiting total knee arthroplasty when compared to men. J. Orthop. Res. Off Publ. Orthop. Res. Soc. 23, 397-405.

Pfeilschifter, J., Seyedin, S.M. and Mundy, G.R. 1988. Transforming growth factor beta inhibits bone resorption in fetal rat long bone cultures. J. Clin. Invest. 82, 680-685.

Ping-Ke, F., Xu-Chen, M., Da-Long, M. and Kai-Yuan, F. 1999. Determination of interleukin-1 receptor antagonist, interleukin-10, and transforming growth factor- $\beta 1$ in synovial fluid aspirates of patients with temporomandibular disorders. J. Oral Maxillofac. Surg. 57, 922-928.

Pond, M.J. and Campbell, J.R. 1972. The canine stifle joint. I. Rupture of the anterior cruciate ligament. An assessment of conservative and surgical treatment. J. Small Anim. Pract. 13, 1-10.

Redini, F., Galera, P., Mauviel, A., Loyau, G. and Pujol, J.P. 1988. Transforming growth factor beta stimulates collagen and glycosaminoglycan biosynthesis in cultured rabbit articular chondrocytes. FEBS Lett. 234, 172-176.

Solimeno, L., Goddard, N., Pasta, G., Mohanty, S., Mortazavi, S., Pacheco, L., Sohail, T. and Luck, J. 2010. Management of arthrofibrosis in haemophilic arthropathy. Haemoph. Off J. World Fed. Hemoph. 16(Suppl. 5), 115-120.

Stern, R., Asari, A.A. and Sugahara, K.N. 2006. Hyaluronan fragments: an information-rich system. Eur. J. Cell Biol. 85, 699-715.

Tanimoto, K., Suzuki, A., Ohno, S., Honda, K., Tanaka, N., Doi, T., Yoneno, K., Ohno-Nakahara, M., Nakatani, Y., Ueki, M. and Tanne, K. 2004. Effects of TGF-beta on hyaluronan anabolism in fibroblasts derived from the synovial membrane of the rabbit temporomandibular joint. J. Dent. Res. 83, 40-44.

Tirgari, M. and Vaughan, L.C. 1975. Arthritis of the canine stifle joint. Vet. Rec. 96, 394-399.

van Beuningen, H.M., Glansbeek, H.L., van der Kraan, P.M. and van den Berg, W.B. 2000. Osteoarthritislike changes in the murine knee joint resulting from intra-articular transforming growth factor-beta injections. Osteoarthritis Cartilage 8, 25-33.

van Beuningen, H.M., van der Kraan, P.M., Arntz, O.J. and van den Berg, W.B. 1994. Transforming growth factor-beta 1 stimulates articular chondrocyte proteoglycan synthesis and induces osteophyte 
formation in the murine knee joint. Lab. Investig. J. Tech. Methods Pathol. 71, 279-290.

Varga, J., Rosenbloom, J. and Jimenez, S.A. 1987. Transforming growth factor beta (TGF beta) causes a persistent increase in steady-state amounts of type I and type III collagen and fibronectin mRNAs in normal human dermal fibroblasts. Biochem. J. 247, 597-604.

Vavken, P., Leumann, A., Hügle, T., Valderrabano, V. and Pagenstert, G. 2013. Kreuzband und Arthrose: Was können wir aus den Tiermodellen lernen, und was ist die klinische Evidenz beim Menschen. Schweiz Z. Für Sportmed. Sporttraumatologie 61, 21-24.

Verdier, M.-P., Seité, S., Guntzer, K., Pujol, J.-P. and Boumédiène, K. 2005. Immunohistochemical analysis of transforming growth factor beta isoforms and their receptors in human cartilage from normal and osteoarthritic femoral heads. Rheumatol. Int. 2, 118-124.

Wahl, S.M., Allen, J.B., Costa, G.L., Wong, H.L., Dasch, J.R. 1993. Reversal of acute and chronic synovial inflammation by anti-transforming growth factor beta. J. Exp. Med. 177, 225-230.

Wynn, T. 2008. Cellular and molecular mechanisms of fibrosis. J. Pathol. 214, 199-210.

Yang, X., Chen, L., Xu, X., Li, C., Huang, C. and Deng, C.X. 2001. TGF-beta/Smad3 signals repress chondrocyte hypertrophic differentiation and are required for maintaining articular cartilage. J. Cell Biol. 153, 35-46.

Zeichen, J., Haeder, L., Jagodzinski, M., Lobenhoffer, P., Bosch, U. and Brand, J. 2008. Localisation of TGF-beta and PDGF and their relevance for the pathogenesis of arthrofibrosis. Unfallchirurg 111, 79-84. 